CLINICAL STUDY

\title{
Bone mineral density, bone markers, and fractures in adult males with congenital adrenal hyperplasia
}

\author{
Henrik Falhammar ${ }^{1,2}$, Helena Filipsson Nyström ${ }^{3}$, Anna Wedell ${ }^{2,4}$, Kerstin Brismar ${ }^{1,2}$ and Marja Thorén ${ }^{1,2}$ \\ ${ }^{1}$ Department of Endocrinology, Metabolism and Diabetes, D02:04, Karolinska University Hospital, SE-171 76 Stockholm, Sweden, ${ }^{2}$ Department of \\ Molecular Medicine and Surgery, Karolinska Institutet, Stockholm, Sweden, ${ }^{3}$ Department of Endocrinology, Sahlgrenska University Hospital and \\ Sahlgrenska Academy, University of Gothenburg, Göteborg, Sweden and ${ }^{4}$ Centre for Inherited Metabolic Diseases (CMMS), Karolinska University Hospital, \\ Stockholm, Sweden
}

(Correspondence should be addressed to H Falhammar at Department of Endocrinology, Metabolism and Diabetes, D02:04, Karolinska University Hospital; Email: henrik.falhammar@ki.se)

\begin{abstract}
Objective: The aim of this study was to determine bone mineral density (BMD), markers of bone metabolism, fractures, and steroids reflecting hormonal control in adult males with congenital adrenal hyperplasia (CAH).

Subjects, methods, and design: We compared CAH males with 21-hydroxylase deficiency $(n=30), 19-67$ years old, with age- and sex-matched controls $(n=32)$. Subgroups of CYP21A2 genotypes, age, glucocorticoid preparation, poor control vs overtreatment, and early vs late ( $>36$ months) diagnosis were studied. BMD measured by dual energy X-ray absorptiometry and markers of bone metabolism and androgens/17-hydroxyprogesterone levels were investigated.

Results: All, including older ( $>30$ years), CAH patients had lower BMD in all measured sites compared with control subjects. The null group demonstrated lower BMD in more locations than the other groups. Osteoporosis/osteopenia was present in $81 \%$ of CAH patients compared with $32 \%$ in controls ( $\geq 30$ years). Fracture frequency was similar, osteocalcin was lower, and fewer patients than controls had vitamin D insufficiency. IGF1 was elevated in the milder genotypes. In patients, total body BMD was positively correlated to weight, BMI, total lean body mass, and triglycerides, and negatively to prolactin. Patients on prednisolone had lower BMD and osteocalcin levels than those on hydrocortisone/cortisone acetate. Patients with poor control had higher femoral neck BMD. There were no differences in BMD between patients with an early vs late diagnosis.

Conclusions: CAH males have low BMD and bone formation markers. BMD should be monitored, adequate prophylaxis and treatment established, and glucocorticoid doses optimized to minimize the risk of future fractures.
\end{abstract}

European Journal of Endocrinology 168 331-341

\section{Introduction}

Congenital adrenal hyperplasia $(\mathrm{CAH})$ is characterized by impaired activity of one of the enzymes needed for cortisol synthesis. The most commonly affected enzyme, 21-hydroxylase, results in cortisol and aldosterone deficiency in addition to androgen excess $(1,2)$. Therapy in these cases consists of life-long glucocorticoid and often mineralocorticoid supplementation that will compensate the cortisol deficiency and normalize the elevated androgens. However, oral glucocorticoid replacement cannot accomplish normal variations in serum cortisol and the doses needed to suppress adrenal androgens are often somewhat supraphysiological. It is very well established that glucocorticoid excess in endogenous Cushing's syndrome and pharmacological glucocorticoid therapy can lead to osteoporosis via multiple mechanisms by increasing bone resorption and decreasing bone formation. Intestinal calcium absorption may also be impaired and renal calcium excretion enhanced leading to secondary hyperparathyroidism. IGF1, their binding proteins and the secretion of gonadal steroids can also be affected (3).

However, reported data concerning bone mass and bone metabolism in CAH patients are conflicting. In children and young adults, bone mineral density (BMD) was reported to be increased (4), normal $(5,6)$, or decreased (7). Other pediatric studies found decreased BMD in males (8), in long-term treated girls (9), and at puberty (10). In adults, BMD has been shown to be normal $(11,12,13,14)$, or decreased $(15,16,17,18,19,20,21,22,23,24)$. Moreover, we have previously described increased fracture frequency compared with age- and sex-matched controls in $\mathrm{CAH}$ women (19). Therefore, we wanted to explore bone health in adult $\mathrm{CAH}$ males. Furthermore, the secretion 
of gonadal steroids is an important determinant of bone development and is very different in the two sexes.

The aim of this study was to evaluate bone health in adult men with $\mathrm{CAH}$ in more detail. We investigated BMD, fracture prevalence, markers of bone metabolism, and the relationship of these parameters to the therapy with adrenocortical hormones. We also studied whether there were differences in these parameters in relation to the CYP21A2 genotypes or between younger and older patients. When applicable, the results were compared with those in age- and sex-matched controls.

\section{Materials and methods}

\section{Subjects}

The study group comprised 32 adult males aged 19-67 years $(>40$ years, $n=10 ;>50$ years, $n=5)$ with genetically confirmed $\mathrm{CAH}$. They were recruited from the two participating university hospitals. Male controls, one for each patient and born on the same date as each patient, were recruited from the National Population Registry. Characteristics of this cohort have been reported earlier $(25,26)$.

In brief, 31 patients had 21-hydroxylase deficiency (CYP21A2 gene mutations) and one had 3 $\beta$-hydroxysteroid dehydrogenase (3 $\beta$-HSD) type II deficiency (C75R genotype). The phenotype was classic in 30, and 17 of them had the salt-wasting (SW) form. Two patients had the non-classic (NC) phenotype.

The patient with $3 \beta$-HSD type II deficiency and one male with CYP21A2 gene mutation and female karyotype were excluded from the statistical analyses, but are separately described in the Results section. Thus, 30 patients were included in the statistical analysis.

All subjects were divided into two age groups, $<30$ years (patients $n=9$, controls $n=10$ ) and $\geq 30$ years (patients $n=21$, controls $n=22$ ). Pediatric endocrinology was introduced in Sweden about 30 years before the inclusion of the present cohort, which could affect the outcome. The patient data were also divided into subgroups according to the three most prevalent CYP21A2 mutations: null, I2 splice, and I172N. Null refers to mutations completely abolishing enzyme activity and is associated with the SW phenotype. I2 splice retains a very low, but measurable, level of activity and is usually associated with SW, whereas I172N is less severe and found most often in the simple virilizing (SV) form. Effects of early ( $<36$ months) and late diagnosis ( $\geq 36$ months), and of poor treatment control, arbitrarily defined as urinary pregnanetriol $\geq 110 \mu \mathrm{mol} / 24 \mathrm{~h}$ and/or a 24-h median 17-hydroxyprogesterone $(17 \mathrm{OHP})$ value $\geq 96 \mathrm{nmol} / \mathrm{l}$, and of overtreatment (a 24-h median 170HP value at the level of detection i.e. $\leq 5 \mathrm{nmol} / \mathrm{l}$ ) were also studied.

The study was approved by the Ethics Committees of the Karolinska Institutet, Stockholm, and the University of Gothenburg, Göteborg, Sweden. All participants gave their written informed consent.

\section{Study protocol}

Patients and controls were examined as outpatients at the Department of Endocrinology, Metabolism and Diabetes, Karolinska University Hospital, Stockholm (patients $n=22$, controls $n=22$ ) or the Department of Endocrinology, Sahlgrenska University Hospital, Göteborg (patients $n=10$, controls $n=10$ ), Sweden. Medical histories were obtained from both patients and controls. BMD was studied by dual energy X-ray absorptiometry (DXA). Blood samples were collected after an overnight fast. A morning urinary sample was collected for NTX and creatinine. In patients, a 24-h urinary pregnanetriol and a diurnal 170HP curve using dried blood spots were analyzed.

\section{Bone mineral density}

Total body, lumbar spine (L1-L4) and femoral neck BMD were estimated by DXA (Lunar Model Prodigy equipment; Lunar Radiation, Madison, WI, USA). The instruments at the two investigation sites were calibrated. Results were calculated both in $\mathrm{g} / \mathrm{cm}^{2}$, Z-score, and T-score. A T-score between -1 and -2.5 S.D. from the mean of young male adults at any measured site was defined as osteopenia, and values below -2.5 S.D. as osteoporosis.

\section{Fractures}

All types of clinical fractures which had been verified by X-ray were recorded. Fractures in vertebrae, wrist, and hip were considered to be associated with osteoporosis. The FRAX tool (www.shef.ac.uk/FRAX/) was used to evaluate fracture risk in patients and controls. This instrument provides fracture risk assessments in individuals aged 40 years or older.

\section{Glucocorticoid supplementation}

The current glucocorticoid doses were converted to hydrocortisone equivalents using anti-inflammatory equivalents $(30 \mathrm{mg}$ hydrocortisone $=37.5 \mathrm{mg}$ cortisone acetate $=7.5 \mathrm{mg}$ prednisolone $=0.75 \mathrm{mg}$ dexamethasone) (27). Body surface area was calculated as the square root of (height $(\mathrm{cm}) \times$ weight $(\mathrm{kg})) / 3600\left(\mathrm{~m}^{2}\right)$ and was used to indicate hydrocortisone equivalents in $\mathrm{mg} / \mathrm{m}^{2}$.

\section{Biochemical assays}

All assays were performed on serum unless otherwise stated. Testosterone, estradiol and dried blood spot 170HP (measured at 0800, 1400, 1900, 0100, and 
$0600 \mathrm{~h}$ ), and 24-h urinary pregnanetriol were measured as described previously $(25,26)$.

DHEAS and PTH were measured on an Advantage automatic immune analyzer (Nichols Institute Diagnostics, San Clemente, CA, USA); the reference limits of PTH were 12-55 ng/l. RIA methods were used for the determination of androstenedione (DiaSorin S.p.A., Saluggia, Italy) and IGF1 (28). IGF1 levels expressed as SDS were calculated from values in 448 healthy subjects (29). Carboxy-terminal cross-linked telopeptide of type I collagen (CTX) was measured on a Roche Elecsys 1010/2010 immunoassay analyzer (Roche Diagnostics Ltd.); reference limits were for males $<50$ years $<580 \mathrm{ng} / \mathrm{l}$ and 50-70 years $<700 \mathrm{ng} / \mathrm{l}$. Bonespecific ALP (BALP) was measured with the Access Ostase assay with reference limit in males $<20 \mu \mathrm{g} / \mathrm{l}$, and for prolactin a chemiluminescent immunoassay (Beckman Coulter, Fullerton, CA, USA) was used. Osteocalcin was determined by an IRMA (CIS Bio International, Gif-sur-Yvette, France); reference limits were for males 18-29 years $<70 \mu \mathrm{g} / \mathrm{l}$, 30-49 years $<45 \mu \mathrm{g} / \mathrm{l}$, and $\geq 50$ years $<50 \mu \mathrm{g} / \mathrm{l}$. A chemiluminescent immunoassay (DiaSorin S.p.A.) was used for analyzing 25-hydroxy-vitamin D (250HD); levels $<25 \mathrm{nmol} / \mathrm{l}$ indicated deficiency, 25-74 nmol/l insufficiency, and optimal levels 75-250 nmol/l (30). Urinary amino-terminal collagen crosslinks (NTX) was measured with enzyme immunoassay Osteomark (Inverness Medical, Princeton, NJ, USA); reference limits were 3-51 nmol/l bone collagen equivalents/ mmol creatinine. Ionized calcium, phosphate, albumin, creatinine, and thyroid function tests were determined by standardized and certified procedures. The within and between assay coefficients of variation were PTH, 6.7 and 8.7\%; DHEAS, 4.4 and 8.7\%; IGF1, 4 and $11 \%$; CTX, 2 and 17.9\%; BALP, 4.2 and $10 \%$; osteocalcin, 3.5 and $4.2 \%$; 25OHD, 2.9 and $7.9 \%$; and NTX, 4 and $10 \%$.

\section{Statistical analysis}

Results are presented as the mean \pm s.D. unless otherwise stated. Comparisons between two groups were made using the unpaired $t$-test when values were normally distributed. Otherwise, the Mann-Whitney rank-sum test was used and in these cases the median and range are reported. When continuous variables were compared in three groups, one-way ANOVA was used for normal distributions, otherwise the KruskalWallis test was performed. $\chi^{2}$ was used in frequency table calculations or, when the expected frequency was small $(<5)$ Fisher's exact test was used. All proportions were calculated discounting missing values. Correlations between variables were assessed using linear and multiple regression analysis. Statistical significance was set at $P<0.05$ and tendency at 0.05-0.10. Data were analyzed using SigmaStat for Windows (Systat Software, Inc., San Jose, CA, USA).

\section{Results}

\section{Bone mineral density}

All patients (including the older cohort) with CAH had lower BMD at all measured sites compared with controls (Table 1 and Fig. 1). Older patients had lower BMD in femoral neck compared with younger patients, whereas there were no differences between younger and older control subjects. Osteoporosis or osteopenia was present in $67 \%$ of all $\mathrm{CAH}$ patients compared with $39 \%$ of all controls $(P=0.054)$, and in males $\geq 30$ years the corresponding figures were $81 \%$ compared with $32 \%$ $(P=0.003)$. Compared with controls, total body BMD was significantly lowered in the null and I2 splice genotype groups and showed a similar trend in the I172N group (Table 1). In the null genotype group femoral neck BMD was also decreased (Table 1). Nine patients were on calcium and vitamin D supplementation for osteoporosis or osteopenia compared with none among the controls $(P<0.001)$. Two of these patients, aged 41 and 67 years, had in addition bisphosphonate therapy with alendronate.

\section{Fractures}

The numbers of individuals with fractures (total or associated with osteoporosis) were similar in the entire group and in all subgroups compared with their respective controls (Table 1). No difference between the younger and older groups was found (data not shown). Only one patient $>50$ years had fractures (clavicle and finger). The $1172 \mathrm{~N}$ group had more individuals with a fracture than the null group (Table 1). When the total number of fractures was compared the result was similar between patients and controls (data not shown). The fractures had often occurred during sport activities, but the underlying trauma was not recorded systematically. Calculation of fracture risk indicated that the 10 years, probability of a major osteoporotic fracture was $8.1 \pm 4.0 \%$ in $\mathrm{CAH}$ males compared with $4.9 \pm 3.0 \%$ in controls $(P=0.058)$, and a hip fracture was $2.2 \pm 2.1 \%$ in $\mathrm{CAH}$ males compared with $0.8 \pm 0.7 \%$ in controls $(P=0.050)$. However, if the sole $\mathrm{CAH}$ male patient who did not use glucocorticoids was excluded, FRAX evaluation of risk factor increased slightly to $8.6 \pm 3.8$ and $2.4 \pm 2.1 \% \quad(P=0.025$ and 0.029 respectively compared with controls).

A 23-year-old patient (I2 splice, lumbar spine T-score -1.0 S.D. otherwise normal BMD, $5 \mathrm{mg}$ prednisolone daily, poor control) had 21 individual fractures all obtained during sports activities such as boxing and football. A 41-year-old patient (I2 splice, T-score - 3.5 s.D. in total body, $6.25 \mathrm{mg}$ prednisolone daily, good control, serum testosterone $18 \mathrm{nmol} / \mathrm{l}$, lactose intolerant) had a spontaneous vertebral fracture, and used alendronate in addition to calcium and vitamin D supplementation. 


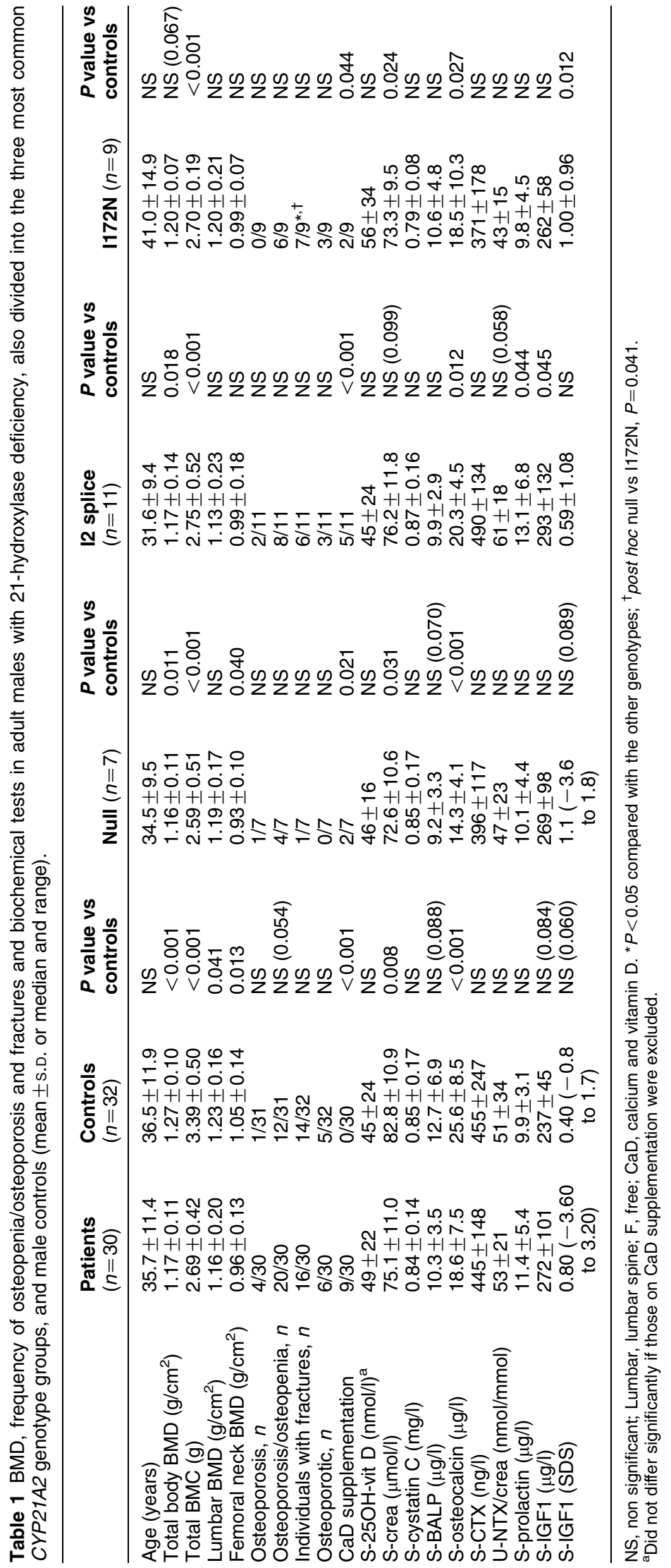




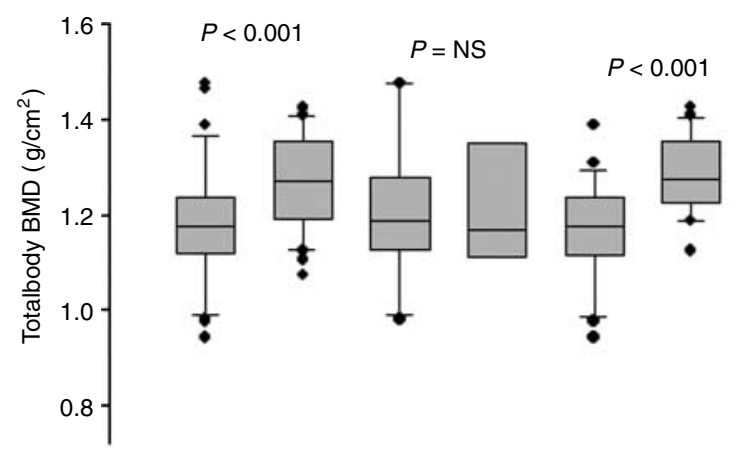

\section{Markers for bone metabolism and other biochemical tests}

Results of biochemical tests are shown in Table 1.

Bone formation markers Serum osteocalcin levels were lower in all cohorts of patients compared with the control group, whereas serum BALP levels tended to be decreased in all patients and in the null genotype group. All values were within normal range except for two subjects in the control group having elevated BALP levels.

Bone resorption markers Urinary NTX tended to be increased in the I2 splice group. Eleven patients and five subjects in the control group had values above the upper reference limit. Serum CTX was not different in all comparisons between patients and control subjects, but was increased in both younger patients and younger control subjects compared with older patients and older control subjects. Seven patients and eight control subjects had concentrations above the upper reference range.

Calcium homeostasis and renal function Ionized calcium and albumin levels tended $(P=0.088$ and 0.060 respectively) to be lower in patients compared with control subjects (data not shown). Three patients and one control subject had modestly elevated PTH levels. Only three patients and three control subjects had optimal vitamin D values $(>75 \mathrm{nmol} / \mathrm{l})$. One of these patients was on calcium and vitamin D supplementation. Fewer $\mathrm{CAH}$ males had vitamin D deficiency $(<25 \mathrm{nmol} / \mathrm{l})$ compared with control subjects (3/30 vs $10 / 28, P=0.042)$. There was no obvious difference between patient and control groups regarding the time of year for blood sample collection. Creatinine was lower in all groups of patients compared with controls (except in younger $\mathrm{CAH}$ males and in $\mathrm{I} 2$ splice it was only a tendency), but serum cystatin $C$ was similar between all groups. PTH and phosphate did not differ between the groups (data not shown).

Nonsteroid hormone determinations IGF1 was elevated in the I2 splice group $(\mu \mathrm{g} / \mathrm{l})$ and in the $\mathrm{I} 172 \mathrm{~N}$ group (SDS) compared with controls. Prolactin was elevated in the I2 splice group compared with the control group. No differences were found in thyroid function tests except for a slight increase in free $\mathrm{T}_{3}$ in the null group (data not shown).

\section{Androgen levels}

Although in many patients androstenedione levels were either higher or lower than in the control subjects, the average level was not different between the two groups. In contrast, in almost all patients DHEAS levels were very low compared with control subjects 

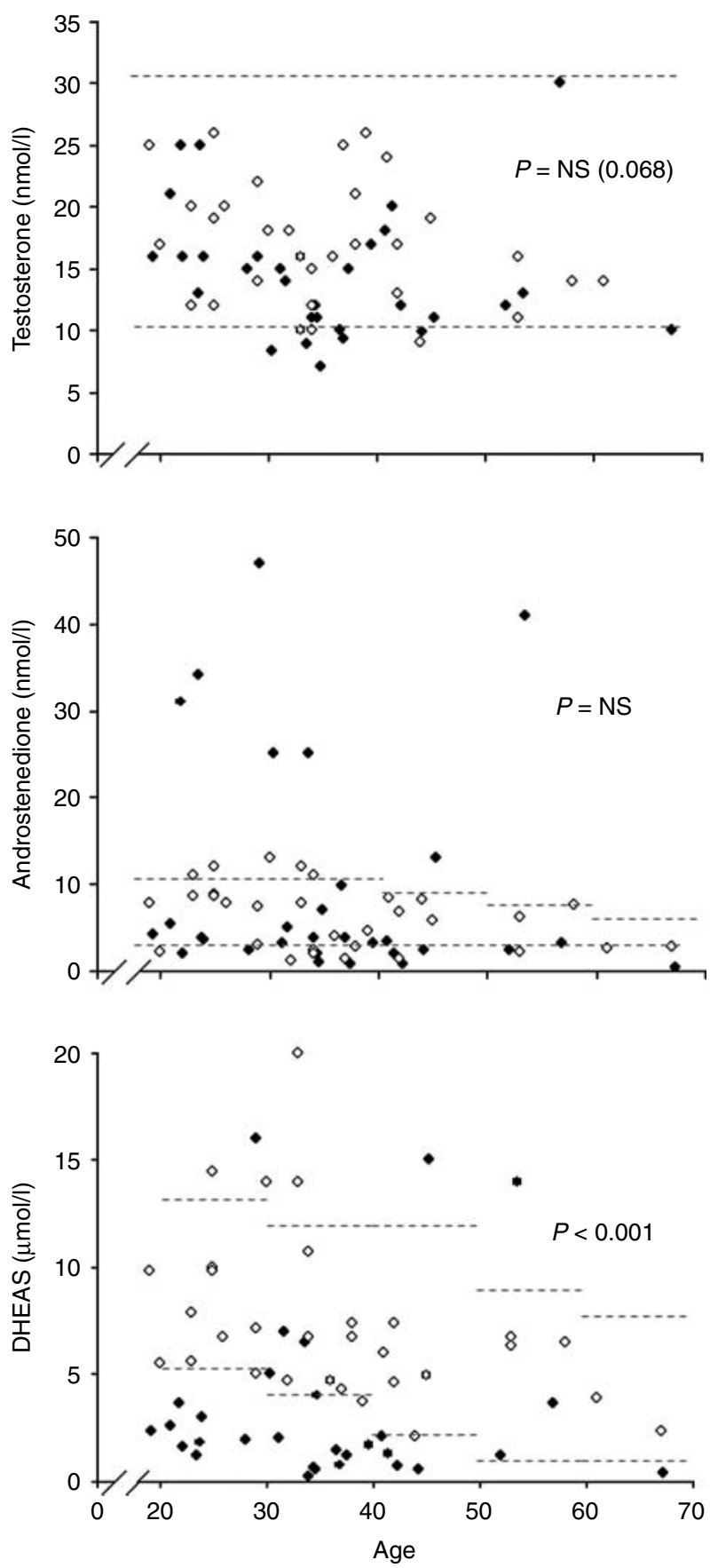

Figure 2 Serum androgens in adult men with 21-hydroxylase deficiency and age- and sex-matched controls. Filled diamonds, $\mathrm{CAH}$ males. Unfilled diamonds, control subjects. Reference limits for the different androgens and different age intervals are indicated with dotted lines. $P$ values indicate the difference between $\mathrm{CAH}$ males and control subjects.

(Fig. 2). Overall, serum testosterone levels in the patients

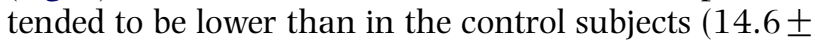
5.4 vs $17.0 \pm 4.9 \mathrm{nmol} / \mathrm{l}, P=0.068)$. The difference was significant in the older group $(13.1 \pm 5.1$ vs $16.2 \pm$ $4.8 \mathrm{nmol} / \mathrm{l}, P=0.045$ ) but not in the younger group
$(16.8 \pm 3.9$ vs $18.7 \pm 5.0 \mathrm{nmol} / \mathrm{l}, P=\mathrm{NS})$ (partly presented in reference (25)).

\section{Corticosteroid therapy and control of treatment}

Glucocorticoids were taken by 93\% (28/30) of the patients $(25,26)$. The most frequently used preparations were prednisolone $(62 \%, 18 / 29)$ and hydrocortisone $(17 \%, 5 / 29)$. The mean dose in hydrocortisone equivalents was $17.4 \pm 5.2 \mathrm{mg} / \mathrm{m}^{2}(32.7 \pm 12.4 \mathrm{mg})$ without differences between younger and older patients or genotype groups. Most patients $(87 \%, 26 / 30)$ received fludrocortisone with a mean dose of $0.11 \pm 0.06 \mathrm{mg}$, with similar doses in the different groups. Decreased BMD and osteocalcin levels compared with control subjects were present in the group of patients treated with prednisolone but not in patients on short-acting glucocorticoids in spite of similar doses in hydrocortisone equivalents. Increased serum IGF1 level was only present in those on short-acting glucocorticoids (Table 2). Androgen levels were not different.

The 170HP curves varied considerably between the individual patients (Fig. 3). Forty-four percent (7/16) of prednisolone-treated patients had no diurnal variations compared with $14 \%(1 / 7)$ of those on hydrocortisone/cortisone acetate $(P=N S)$. The one patient on dexamethasone also lacked diurnal variations. The area under the curve for $170 \mathrm{HP}$ was $1995 \mathrm{nmol} / \mathrm{l} \times \mathrm{h}$ (630-22 650) in patients on prednisolone, $2025 \mathrm{nmol} / \mathrm{l}$ $\times \mathrm{h}(600-16470)$ for those on hydrocortisone/cortisone acetate, and $690 \mathrm{nmol} / \mathrm{l} \times \mathrm{h}$ in one patient on dexamethasone. There were no significant differences between the three genotype groups (not shown).

\section{Poor control vs overtreatment}

Patients with poor control $(n=7)$ had significantly higher femoral neck BMD and a tendency to decreased urinary NTX/creatinine compared with patients having suppressed 170HP and/or urinary pregnanetriol $(n=10)$ ( $T$-score $-0.70 \pm 0.53$ vs $-1.56 \pm 0.75$ SDS, $P=0.025 ; 38 \pm 23$ vs $61 \pm 17 \mathrm{nmol} / \mathrm{mmol}, P=0.056)$. $\mathrm{BMD}$ at other sites did not differ between those with poor control and those with overtreated (total body $T$-score $-0.74 \pm 0.50$ vs $-1.44 \pm 1.03$ SDS; lumbar spine $T$-score $-0.74 \pm 0.47$ vs $-0.49 \pm 2.44$ SDS; osteoporosis/osteopenia $5 / 7$ vs $6 / 10$; fractures $4 / 7$ vs $6 / 10)$. The results were similar if BMD $\left(\mathrm{g} / \mathrm{cm}^{2}\right)$ or Z-score were used (data not shown).

\section{SW vs SV phenotype}

No differences between SW and SV were found in the parameters reported above (data not shown) apart from a tendency to higher urinary NTX in SW compared with $\mathrm{SV}(58 \pm 18$ vs $39 \pm 16 \mathrm{nmol} / \mathrm{l}, P=0.062)$. 
Table 2 BMD, frequency of osteopenia/osteoporosis, fractures and biochemical tests in adult males with 21-hydroxylase deficiency on different glucocorticoids and male controls (mean \pm s.D. or median and range).

\begin{tabular}{|c|c|c|c|c|c|}
\hline & $\begin{array}{l}\text { Prednisolone } \\
(n=18)\end{array}$ & $\begin{array}{l}P \text { value vs } \\
\text { controls }\end{array}$ & $\begin{array}{l}\text { HC or CoAc } \\
(n=8)\end{array}$ & $\begin{array}{l}P \text { value vs } \\
\text { controls }\end{array}$ & $\begin{array}{l}\text { Controls } \\
(n=32)\end{array}$ \\
\hline Age (years) & $35.6 \pm 12.0$ & NS & $37.4 \pm 12.8$ & NS & $36.5 \pm 11.9$ \\
\hline Total body BMD $\left(\mathrm{g} / \mathrm{cm}^{2}\right)$ & $1.13 \pm 0.10$ & $<0.001$ & $1.25 \pm 0.11^{*}$ & NS & $1.27 \pm 0.10$ \\
\hline Total body ( $T$-score) & $-1.25 \pm 1.71$ & $<0.001$ & $-0 . \overline{14} \pm 1.22^{*}$ & NS & $0.53 \pm 1.15$ \\
\hline Total BMC (q) & $2.54+0.49$ & $<0.001$ & $2.88+0.33^{\dagger}$ & 0.009 & $3.39+0.50$ \\
\hline Lumbar BMD (g/cm²) & $1.12 \pm 0.18$ & 0.031 & $1.22 \pm 0.24$ & NS & $1.23 \pm 0.16$ \\
\hline Lumbar ( $T$-score) & $-0.94 \pm 1.44$ & 0.026 & $-0.30 \pm 2.14$ & NS & $-0.01 \pm 1.33$ \\
\hline Femoral neck BMD $\left(\mathrm{g} / \mathrm{cm}^{2}\right)$ & $0.94 \pm 0.12$ & 0.006 & $0.99 \pm 0.17$ & NS & $1.05 \pm 0.14$ \\
\hline Femoral neck ( $T$-score) & $-1.22 \pm 0.84$ & 0.002 & $-0.83 \pm 1.38$ & NS & $-0.21 \pm 1.10$ \\
\hline Osteoporosis & $4 / 18$ & 0.047 & $0 / 8$ & NS & $1 / 31$ \\
\hline Osteoporosis/osteopenia & $12 / 18$ & NS (0.069) & $6 / 8$ & NS & $12 / 31$ \\
\hline Individuals with fractures & $9 / 18$ & NS & $4 / 8$ & NS & $14 / 32$ \\
\hline Osteoporotic & $4 / 18$ & NS & $1 / 8$ & NS & $5 / 32$ \\
\hline $\mathrm{CaD}$ supplementation & $7 / 18$ & 0.001 & $2 / 8$ & 0.036 & $0 / 32$ \\
\hline S-25OH-vit D & $47 \pm 16$ & NS & $55 \pm 31$ & NS & $45 \pm 24$ \\
\hline S-crea $(\mu \mathrm{mol} / \mathrm{l})$ & $73.1 \pm 10.2$ & 0.004 & $79 . \overline{3} \pm 11.9$ & NS & $82 . \overline{8} \pm 10.9$ \\
\hline S-osteocalcin $(\mu \mathrm{g} / \mathrm{l})$ & $16.6+4.6$ & $<0.001$ & $19.5+9.1$ & NS $(0.058)$ & $25.6+8.5$ \\
\hline S-CTX (ng/l) & $426 \pm 129$ & NS & $461 \pm 173$ & NS & $455 \pm 247$ \\
\hline U-NTX/crea & $52+21$ & NS & $56+14$ & NS & $51+34$ \\
\hline S-estradiol $(\mathrm{pmol} / \mathrm{l})$ & $91(26-298)$ & NS & $79(49-169)$ & NS & $75(43-148)$ \\
\hline S-prolactin $(\mu \mathrm{g} / \mathrm{l})$ & $12.1 \pm 5.8$ & NS $(0.091)$ & $10.7 \pm 4.9$ & NS & $9.9 \pm 3.1$ \\
\hline S-IGF1 ( $\mu \mathrm{g} / \mathrm{l})$ & $237 \pm 72$ & NS & $305 \pm 41^{*}$ & $<0.001$ & $237 \pm 45$ \\
\hline S-IGF1 (SDS) & $0.17 \pm 1.20$ & NS & $1.29 \pm 1.01^{*}$ & $<0.001$ & $0.34 \pm 0.54$ \\
\hline $\mathrm{HCeq} / \mathrm{m}^{2}\left(\mathrm{mg} / \mathrm{m}^{2}\right)$ & $16.8 \pm 4.6$ & & $19.9 \pm 6.3$ & & \\
\hline Months on GC & $423(231-635)$ & & $391(284-470)$ & & \\
\hline AUC 17OHP $(\mathrm{nmol} / \mathrm{I} \times \mathrm{h})$ & $1995(630-22650)$ & & $2025(600-16470)$ & & \\
\hline
\end{tabular}

${ }^{\star} P<0.05,{ }^{\dagger} P=0.05-0.099$ compared with the Prednisolone group. NS, not significant; HC, hydrocortisone; CoAC, cortisone acetate; Lumbar, lumbar spine; HCeq, hydrocortisone equivalents; GC, glucocorticoid; AUC, area under the curve.

\section{Relationships between BMD and other parameters}

BMD was correlated with our previously reported data on anthropometric parameters, body composition, metabolic profile, testicular ultrasound, and sperm analysis $(25,26)$. In patients, total body BMD was positively correlated to weight $(r=0.567, P=0.001)$, BMI $(r=0.448, P=0.013)$, total lean body mass $(r=0.665, \quad P<0.001), \quad$ triglycerides $\quad(r=0.412$, $P=0.024)$, and negatively to prolactin $(r=-0.412$, $P=0.012)$, and total sperm count and concentration $(r=-0.544$ and $-0.533 ; P=0.044$ and 0.049 respectively). No correlations were found between BMD and dose of hydrocortisone equivalents $/ \mathrm{m}^{2}$ or other biochemical markers. In multiple linear regression, the strongest correlation with total body BMD was total lean mass and prolactin $(r=0.746, P<0.001)$.

In controls, total body BMD was positively correlated to weight $(r=0.706, P<0.001)$, BMI $(r=0.556, P=0.001)$, waist $(r=0.433, P=0.027)$, hip $(r=0.650, P<0.001)$, total fat and lean body mass $(r=0.414, P=0.023$ and $r=0.675, P<0.001$ respectively $)$, osteocalcin $(r=0.412$, $P=0.024)$, calcium $(r=0.448, P=0.013)$, and testosterone/estradiol ratio $(r=0.414, P=0.026)$. In multiple linear regression, the strongest correlation with total body BMD was weight and osteocalcin $(r=0.797, P<0.001)$.

Total body BMD was positively correlated to lumbar spine and femoral neck BMD: CAH males $r=0.434$, $P=0.017$ and $r=0.716, P<0.001$; controls $r=0.841$ and $r=0.741$, both $P<0.001$. In patients, lumbar spine BMD was also correlated positively with fludrocortisone dose $(r=0.382, P=0.037)$ and negatively with total functional testicular volume $(r=-0.434, P=0.019)$.

\section{Cases}

A 61-year-old male with karyotype 46,XX (I2 splice) was diagnosed and received glucocorticoids at age 7 , and initiated testosterone replacement at 12 years. Current doses were prednisolone $6.25 \mathrm{mg}$ daily and testosterone $250 \mathrm{mg}$ i.m. every 3rd week. DXA measurement using the male reference range suggested good BMD (T-score lumbar spine 0.6 and femoral neck -0.1 SDS). His 170HP levels indicated oversubstitution.

The 22-year-old patient, with $3 \beta$-HSD type II deficiency, used prednisolone $7.5 \mathrm{mg}$ daily and had good BMD (T-score lumbar spine 0.7 and femoral neck 1.3 SDS). His DHEAS level was high. None of them reported any fractures.

\section{Discussion}

This is a comprehensive study reporting on BMD and markers of bone metabolism in adult $\mathrm{CAH}$ males. We found reduced BMD and almost threefold increase in osteopenia/osteoporosis in patients older than 30 years 
compared with age-matched controls. Biochemical tests indicated suppressed bone formation and a tendency to increased bone resorption. There was no evidence of vitamin D deficiency. The frequency of fractures was not increased but FRAX evaluation indicated increased
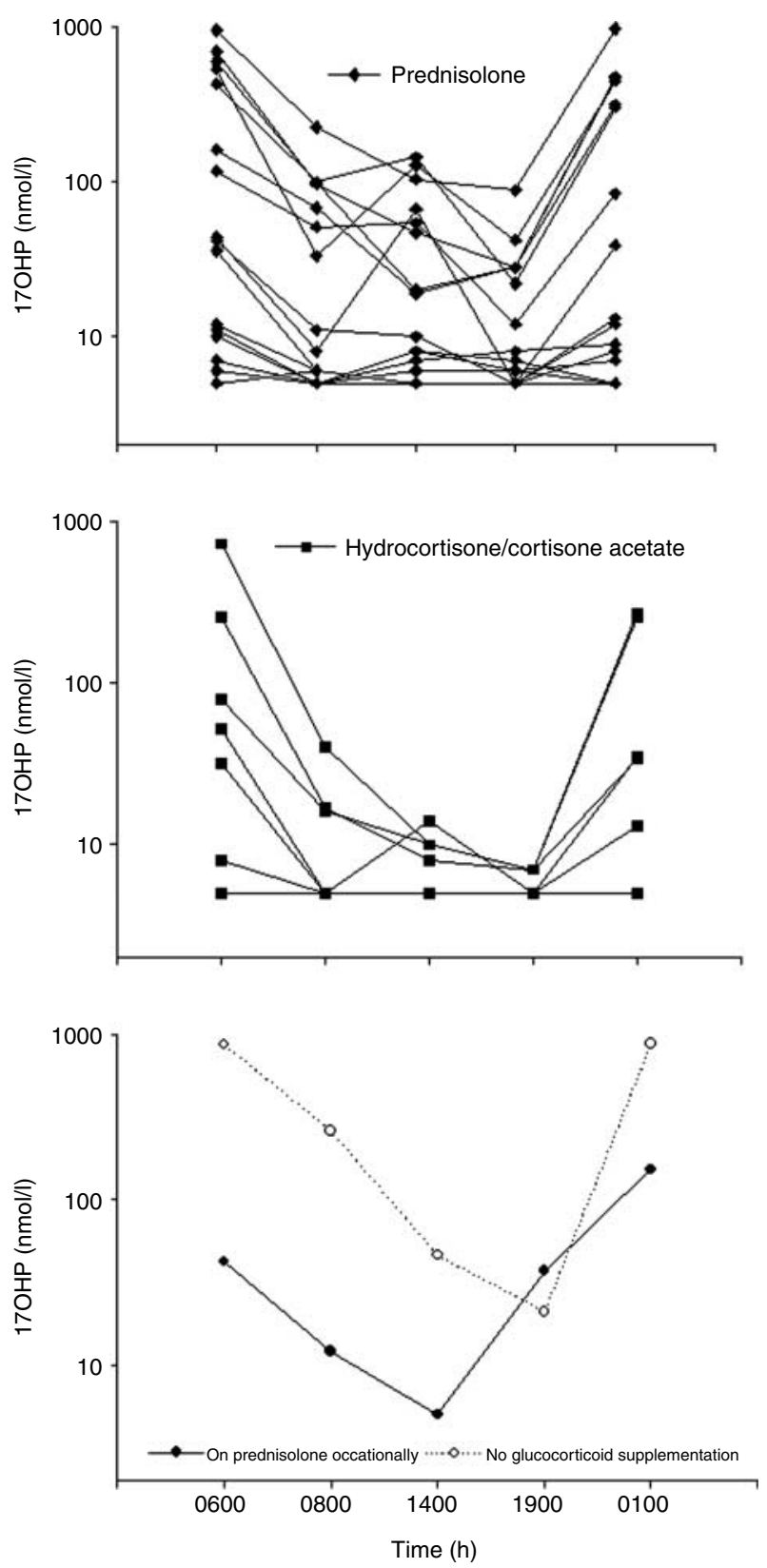

Figure 3 Diurnal 17-hydroxyprogesterone (17OHP) curve analyzed from dried blood spots in adult males with 21-hydroxylase deficiency. Upper panel shows prednisolone supplementation, middle panel shows hydrocortisone/cortisone acetate supplementation, and lower panel shows patients with no regular glucocorticoid supplementation. The 17OHP curve of the male with dexamethasone supplementation is not illustrated; all values were at or less than the level of detection $(5 \mathrm{nmol} / \mathrm{l})$. fracture risk in $\mathrm{CAH}$ males on glucocorticoid supplementation. Bone health appeared less affected in patients taking short-acting glucocorticoids than in those taking more long-acting preparations.

Among our $\mathrm{CAH}$ male patients, $67 \%$ had osteoporosis/osteopenia according to the WHO criteria. Only a few studies report BMD in adult CAH individuals with the results for men and women demonstrated separately $(13,14,17,22,23)$. Our finding of a low BMD is in agreement with the results in some $(17,22,23)$, but not all $(13,14)$, previous studies. In a recent report from Norway, 23 males were included who had a similar age and glucocorticoid doses as our cohort and the frequency of osteopenia/osteoporosis was $57 \%$ (23). Compared with controls, our patients had decreased bone formation markers and similar resorption markers indicating a negative balance with risk for further decrease of BMD. Instead, Sciannamblo et al. (17) found a higher bone turnover than in controls, studying a cohort of $\mathrm{CAH}$ males where some patients were still growing, while a smaller $\mathrm{CAH}$ study with extreme heterogeneity demonstrated decrease of both formation and resorption markers (11).

In spite of the difference in BMD between our patients and control subjects, the frequency of fractures did not differ. Regrettably, we did not ask the source of the trauma that had caused the fractures, and the proportions of low-energy fractures in patients and control subjects are unknown. The patients, however, often spontaneously informed us that the fractures had occurred during sports activities. The only previous report on fractures is from Koetz et al. (22) who studied bone health in primary adrenocortical insufficiency and found only one fracture in $14 \mathrm{CAH}$ males. However, BMD decreases by age in the general population and is associated with increasing fracture risk, and the peak incidence of osteoporotic fractures occurs in old age in males. In our patients, the femoral neck BMD was lower in older than in younger patients. Assessment of fracture risk in our patients of age 40 years and older using the FRAX tool indicated an increased fracture risk. Moreover, it can be assumed that FRAX underestimates the fracture risk as it has not been developed for life-long glucocorticoid supplementation but only calculates glucocorticoid use for more than 3 months as a risk factor. It is of concern that a 41-year-old CAH male with osteoporosis had obtained a spontaneous vertebral fracture. The patient was, however, lactose intolerant which might have contributed to the poor bone health.

Fractures were more prevalent in the milder genotype group I172N compared with the null group despite the fact that BMD at all sites and the mean present glucocorticoid and mineralocorticoid doses were similar (25). A possible explanation for the increased fracture frequency in the I1 $72 \mathrm{~N}$ group might be that the doses of corticosteroids were too high considering the milder disease. We have previously found that the I1 $72 \mathrm{~N}$ group 
also had a more unfavorable cardiovascular/metabolic profile (25).

A positive finding was that younger $\mathrm{CAH}$ males $(<30$ years) had similar BMD to the control subjects. Hopefully, this means that younger $\mathrm{CAH}$ patients have a lower risk of future fractures. The reason for the normal BMD may be improved care during childhood resulting in increased peak bone mass.

An adequate supply of vitamin $D$ is essential for bone health. Studies on $250 H D$ levels in $\mathrm{CAH}$ patients are rare and have shown subnormal values $(22,23)$, while in this study $250 \mathrm{HD}$ did not differ from age- and sex-matched controls. This is in accordance with a recent report showing values in adult classic $\mathrm{CAH}$ similar to the overall population (24). Prescribing vitamin D should be considered in $\mathrm{CAH}$ patients, especially in countries with high risk for low 250HD and osteoporosis, such as Sweden.

As described previously $(19,22)$, we found no relationship between BMD and current glucocorticoid dose. However, correlations between BMD and cumulative glucocorticoid doses have been demonstrated previously $(16,31)$. We found in multiple regression analysis that the strongest correlation with BMD was with lean body mass and prolactin. Prolactin levels have shown to be negatively correlated to BMD in males and in about half of the studies there was no correlation with testosterone levels (32). Prolactin may possibly affect BMD by inducing some degree of hypogonadism but may also have a direct effect on bone.

The most important issue for bone health in the male patients with $\mathrm{CAH}$ is to optimize the glucocorticoid therapy. The ideal is to establish normal diurnal cortisol values and androgen concentrations. In this study, a number of patients had suppressed diurnal 170HP curves without diurnal variation as well as variable reduction of androgen levels indicating that the glucocorticoid doses could be reduced. However, some patients demonstrated very high levels of DHEAS, androstenedione, and diurnal 17OHP. These patients with poor control had better BMD, probably due to longterm high-androgen concentrations and lower exposure to glucocorticoids. We also report herein BMD levels in a patient with $3 \beta$-HSD type II deficiency for the first time. He had a good BMD and the high levels of DHEAS most likely contributed to the favorable outcome. We for the first time also studied BMD in a CAH male with karyotype 46,XX. He had normal values according to the male reference population despite modest overtreatment with glucocorticoids, probably due to regular testosterone replacement since puberty.

The glucocorticoid preparations used varied. Hydrocortisone is the preferred glucocorticoid in children while prednisolone is the preferred option in many adults (2). All our patients with osteoporosis (aged 24-52 years) and the patient with the most serious fracture, a spontaneous vertebrae fracture, used prednisolone. This is in accordance with Jaaskelainen et al. (15), who reported that adult CAH patients substituted with hydrocortisone had better BMD than those on prednisone, prednisolone, or dexamethasone, and the recent study by Koetz et al. (22) describing that hydrocortisone-treated patients with primary adrenal insufficiency had better BMD compared with prednisolone-treated patients. A number of circumstances may contribute to a more negative effect on bone health with prednisolone than with short-acting glucocorticoids. Prednisolone-treated patients received a dose in the evening to suppress the early morning rise of ACTH, $17 \mathrm{OHP}$, and androgens. This may result in a supraphysiological glucocorticoid exposure to the skeleton during late night and early morning hours with a negative impact on BMD. About $40 \%$ of our patients on prednisolone lacked diurnal variations of $17 \mathrm{OHP}$.

Moreover, in our cohort, we have previously found that fat mass was increased compared with controls in hydrocortisone/cortisone acetate-treated patients but not in prednisolone treated (25). It is generally accepted that an increased fat mass is beneficial for the skeleton (33).

IGF1 is a growth factor important for bone tissue. Hydrocortisone/cortisone acetate-treated patients had higher IGF1 SDS compared with controls. This difference was not found in prednisolone-treated patients. It can be speculated that the increased IGF1 might be advantageous. We also found elevated IGF1 levels in adult CAH females (19). It has also been reported in endogenous Cushing's syndrome (34), and in pharmacological glucocorticoid therapy $(35,36)$. Glucocorticoids probably stimulate IGF1 and the extent may vary with glucocorticoid dose and preparation. There is certainly a need for further studies on which glucocorticoid to choose in adult CAH patients for optimal balance between beneficial effects and adverse effects.

The outcome with regard to bone health in $\mathrm{CAH}$ males differs from our previous results in female $\mathrm{CAH}$ patients. Although the reduction in bone density and the frequency of osteopenia/osteoporosis were similar in males and females, the number of fractures differed. As expected, both $\mathrm{CAH}$ males and their controls had more fractures than their female counterparts in line with the higher prevalence of fractures in males than in females before the age of $50-55$ years $(37,38)$. The difference has been attributed to dissimilarities in lifestyle, with males in general having more risky behavior/more participation in rough sports compared with females. However, the fracture frequency was almost ten times increased in $\mathrm{CAH}$ females compared with their controls (30 vs 3\%), whereas there was no difference between CAH males and controls (53 vs 44\%). Although many women with CAH are involved in rough sports (39), it is unlikely to be the only explanation for this outcome. Other circumstances can also be important for the gender difference, for instance compliance with treatment and the dissimilarities in sexual hormone 
concentrations. Moreover, the sensitivity of the glucocorticoid receptors to endogenous and exogenous glucocorticoids is highly individual (40).

The Endocrine Society clinical practice guidelines recommend against the routine evaluation of BMD in children and do not recommend regular monitoring of BMD in adult CAH patients (41). Our findings of low $\mathrm{BMD}$ in $\mathrm{CAH}$ males and previously in $\mathrm{CAH}$ females emphasize the importance of monitoring BMD in at least adult patients starting from young adult age. We have recently recommended DXA measurements of BMD to be done when the patient is transferred from pediatric to adult care and, depending on the BMD levels and the patient's individual risk, repeated every second to fifth year (2). Osteoporosis prophylaxis with recommended physical activities, appropriate nutrition, calcium and vitamin D supplementation, and bisphophonates may be considered. The primary option is, however, optimizing the glucocorticoid supplementation.

The major limitation of this study is its sample size despite being larger than most and including older $\mathrm{CAH}$ males and age- and sex-matched controls in contrast to most other studies. Negative findings must therefore be interpreted with caution. Another limitation is assessing the impact of steroid treatment. Neither type of steroids previously used nor their cumulative lifetime doses were available and we used the dose of the present steroid in the calculations. Steroid excess at an early age may certainly have a continuing negative impact on bone health in adult age.

In conclusion, adult $\mathrm{CAH}$ males with 21-hydroxylase deficiency have decreased BMD and bone formation markers compared with age- and sex-matched controls. Genotype and age at diagnosis did not affect these values. Fractures, bone resorption markers, and vitamin D did not differ between groups. However, overtreatment with glucocorticoids and prednisolone treatment appeared to affect BMD more negatively than poor control and short-acting glucocorticoids.

\section{Declaration of interest}

The authors declare that there is no conflict of interest that could be perceived as prejudicing the impartiality of the research reported.

\section{Funding}

This study was supported by the Magn. Bergvalls Foundation, Swedish Endocrine Society, Karolinska Institutet, Stockholm County Council, and Göteborg Medical Society.

\section{Acknowledgements}

The authors thank Anette Härström and Ingrid Hansson for their excellent care of patients and controls. They also want to thank Sue Maxwell for her exceptional linguistic help in the preparation of this article.

\section{References}

1 Merke DP \& Bornstein SR. Congenital adrenal hyperplasia. Lancet 2005365 2125-2136. (doi:10.1016/S0140-6736(05) 66736-0)

2 Falhammar H \& Thoren M. Clinical outcomes in the management of congenital adrenal hyperplasia. Endocrine 201241 355-373. (doi:10.1007/s12020-011-9591-x)

3 Shaker JL \& Lukert BP. Osteoporosis associated with excess glucocorticoids. Endocrinology and Metabolism Clinics of North America 200534 341-356, viii-ix. (doi:10.1016/j.ecl.2005.01.014)

4 Arisaka O, Hoshi M, Kanazawa S, Numata M, Nakajima D, Kanno S, Negishi M, Nishikura K, Nitta A, Imataka M et al. Preliminary report: effect of adrenal androgen and estrogen on bone maturation and bone mineral density. Metabolism 200150 377-379. (doi:10.1053/meta.2001.21678)

5 Girgis R \& Winter JS. The effects of glucocorticoid replacement therapy on growth, bone mineral density, and bone turnover markers in children with congenital adrenal hyperplasia. Journal of Clinical Endocrinology and Metabolism 199782 3926-3929. (doi:10.1210/jc.82.12.3926)

6 Fleischman A, Ringelheim J, Feldman HA \& Gordon CM. Bone mineral status in children with congenital adrenal hyperplasia. Journal of Pediatric Endocrinology and Metabolism 200720 227-235. (doi:10.1515/JPEM.2007.20.2.227)

7 Zimmermann A, Sido PG, Schulze E, Al Khzouz C, Lazea C, Coldea C \& Weber MM. Bone mineral density and bone turnover in Romanian children and young adults with classical 21-hydroxylase deficiency are influenced by glucocorticoid replacement therapy. Clinical Endocrinology 200971 477-484. (doi:10.1111/ j.1365-2265.2008.03518.x)

8 Cameron FJ, Kaymakci B, Byrt EA, Ebeling PR, Warne GL \& Wark JD. Bone mineral density and body composition in congenital adrenal hyperplasia. Journal of Clinical Endocrinology and Metabolism 1995 80 2238-2243. (doi:10.1210/jc.80.7.2238)

9 de Almeida Freire PO, de Lemos-Marini SH, Maciel-Guerra AT, Morcillo AM, Matias Baptista MT, de Mello MP \& Guerra G Jr. Classical congenital adrenal hyperplasia due to 21-hydroxylase deficiency: a cross-sectional study of factors involved in bone mineral density. Journal of Bone and Mineral Metabolism 200321 396-401. (doi:10.1007/s00774-003-0434-6)

10 Paganini C, Radetti G, Livieri C, Braga V, Migliavacca D \& Adami S. Height, bone mineral density and bone markers in congenital adrenal hyperplasia. Hormone Research $2000 \mathbf{5 4}$ 164-168. (doi:10.1159/000053253)

11 Guo CY, Weetman AP \& Eastell R. Bone turnover and bone mineral density in patients with congenital adrenal hyperplasia. Clinical Endocrinology 199645 535-541. (doi:10.1046/j.13652265.1996.00851.x)

12 Mora S, Saggion F, Russo G, Weber G, Bellini A, Prinster C \& Chiumello G. Bone density in young patients with congenital adrenal hyperplasia. Bone 199618 337-340. (doi:10.1016/ 8756-3282(96)00003-8)

13 Stikkelbroeck NM, Oyen WJ, van der Wilt GJ, Hermus AR \& Otten BJ. Normal bone mineral density and lean body mass, but increased fat mass, in young adult patients with congenital adrenal hyperplasia. Journal of Clinical Endocrinology and Metabolism 200388 1036-1042. (doi:10.1210/jc.2002-021074)

14 Christiansen P, Molgaard C \& Muller J. Normal bone mineral content in young adults with congenital adrenal hyperplasia due to 21-hydroxylase deficiency. Hormone Research 200461 133-136. (doi:10.1159/000075588)

15 Jaaskelainen J \& Voutilainen R. Bone mineral density in relation to glucocorticoid substitution therapy in adult patients with 21-hydroxylase deficiency. Clinical Endocrinology 199645 707-713. (doi:10.1046/j.1365-2265.1996.8620871.x)

16 Hagenfeldt K, Martin Ritzen E, Ringertz H, Helleday J \& Carlstrom $\mathrm{K}$. Bone mass and body composition of adult women 
with congenital virilizing 21-hydroxylase deficiency after glucocorticoid treatment since infancy. European Journal of Endocrinology 2000143 667-671. (doi:10.1530/eje.0.1430667)

17 Sciannamblo M, Russo G, Cuccato D, Chiumello G \& Mora S. Reduced bone mineral density and increased bone metabolism rate in young adult patients with 21-hydroxylase deficiency. Journal of Clinical Endocrinology and Metabolism 200691 4453-4458. (doi:10.1210/jc.2005-2823)

18 King JA, Wisniewski AB, Bankowski BJ, Carson KA, Zacur HA \& Migeon CJ. Long-term corticosteroid replacement and bone mineral density in adult women with classical congenital adrenal hyperplasia. Journal of Clinical Endocrinology and Metabolism 2006 91 865-869. (doi:10.1210/jc.2005-0745)

19 Falhammar H, Filipsson H, Holmdahl G, Janson PO, Nordenskjold A, Hagenfeldt K \& Thoren M. Fractures and bone mineral density in adult women with 21-hydroxylase deficiency. Journal of Clinical Endocrinology and Metabolism 2007 92 4643-4649. (doi:10.1210/jc.2007-0744)

20 Bachelot A, Chakhtoura Z, Rouxel A, Dulon J \& Touraine P. Hormonal treatment of congenital adrenal hyperplasia due to 21-hydroxylase deficiency. Annales d'Endocrinologie $2007 \mathbf{6 8}$ 274-280. (doi:10.1016/j.ando.2007.06.019)

21 Arlt W, Willis DS, Wild SH, Krone N, Doherty EJ, Hahner S, Han TS, Carroll PV, Conway GS, Rees DA et al. Health status of adults with congenital adrenal hyperplasia: a cohort study of 203 patients. Journal of Clinical Endocrinology and Metabolism 201095 5110-5121. (doi:10.1210/jc.2010-0917)

22 Koetz KR, Ventz M, Diederich S \& Quinkler M. Bone mineral density is not significantly reduced in adult patients on low-dose glucocorticoid replacement therapy. Journal of Clinical Endocrinology and Metabolism 201297 85-92. (doi:10.1210/jc. 2011-2036)

23 Nermoen I, Bronstad I, Fougner KJ, Svartberg J, Oksnes M, Husebye ES \& Lovas K. Genetic, anthropometric and metabolic features of adult Norwegian patients with 21-hydroxylase deficiency. European Journal of Endocrinology 2012167 507-516. (doi:10.1530/EJE-12-0196)

24 Finkielstain GP, Kim MS, Sinaii N, Nishitani M, Van Ryzin C, Hill SC, Reynolds JC, Hanna RM \& Merke DP. Clinical characteristics of a cohort of 244 patients with congenital adrenal hyperplasia. Journal of Clinical Endocrinology and Metabolism 2012 97 4429-4438. (doi:10.1210/jc.2012-2102)

25 Falhammar H, Filipsson Nystrom H, Wedell A \& Thoren M. Cardiovascular risk, metabolic profile, and body composition in adult males with congenital adrenal hyperplasia due to 21-hydroxylase deficiency. European Journal of Endocrinology 2011164 285-293. (doi:10.1530/EJE-10-0877)

26 Falhammar H, Nystrom HF, Ekstrom U, Granberg S, Wedell A \& Thoren M. Fertility, sexuality and testicular adrenal rest tumors in adult males with congenital adrenal hyperplasia. European Journal of Endocrinology 2012166 441-449. (doi:10.1530/EJE11-0828)

27 Liddle GW. Clinical pharmacology of the anti-inflammatory steroids. Clinical Pharmacology and Therapeutics 19612 615-635.

28 Bang P, Eriksson U, Sara V, Wivall IL \& Hall K. Comparison of acid ethanol extraction and acid gel filtration prior to IGF-I and IGF-II radioimmunoassays: improvement of determinations in acid ethanol extracts by the use of truncated IGF-I as radioligand. Acta Endocrinologica 1991124 620-629.
29 Hilding A, Hall K, Wivall-Helleryd IL, Saaf M, Melin AL \& Thoren M. Serum levels of insulin-like growth factor I in 152 patients with growth hormone deficiency, aged 19-82 years, in relation to those in healthy subjects. Journal of Clinical Endocrinology and Metabolism 199984 2013-2019. (doi:10.1210/jc. 84.6.2013)

30 Holick MF. Vitamin D deficiency. New England Journal of Medicine 2007357 266-281. (doi:10.1056/NEJMra070553)

31 Chakhtoura Z, Bachelot A, Samara-Boustani D, Ruiz JC, Donadille B, Dulon J, Christin-Maitre S, Bouvattier C, Raux-Demay MC, Bouchard $\mathrm{P}$ et al. Impact of total cumulative glucocorticoid dose on bone mineral density in patients with 21-hydroxylase deficiency. European Journal of Endocrinology 2008158 879-887. (doi:10.1530/EJE-07-0887)

32 Shibli-Rahhal A \& Schlechte J. The effects of hyperprolactinemia on bone and fat. Pituitary 200912 96-104. (doi:10.1007/ s11102-008-0097-3)

33 Zaidi M, Buettner C, Sun L \& Iqbal J. Minireview: the link between fat and bone: does mass beget mass? Endocrinology 2012153 2070-2075. (doi:10.1210/en.2012-1022)

34 Bang P, Degerblad M, Thoren M, Schwander J, Blum W \& Hall K. Insulin-like growth factor (IGF) I and II and IGF binding protein (IGFBP) 1, 2 and 3 in serum from patients with Cushing's syndrome. Acta Endocrinologica 1993128 397-404.

35 Zhou X, Loke KY, Pillai CC, How HK, Yap HK \& Lee KO. IGFs and IGF-binding proteins in short children with steroid-dependent nephrotic syndrome on chronic glucocorticoids: changes with 1 year exogenous GH. European Journal of Endocrinology 2001144 237-243. (doi:10.1530/eje.0.1440237)

36 Short KR, Nygren J, Bigelow ML \& Nair KS. Effect of short-term prednisone use on blood flow, muscle protein metabolism, and function. Journal of Clinical Endocrinology and Metabolism 200489 6198-6207. (doi:10.1210/jc.2004-0908)

37 Donaldson LJ, Reckless IP, Scholes S, Mindell JS \& Shelton NJ. The epidemiology of fractures in England. Journal of Epidemiology and Community Health 200862 174-180. (doi:10.1136/jech.2006. 056622)

38 Khosla S, Amin S \& Orwoll E. Osteoporosis in men. Endocrine Reviews 200829 441-464. (doi:10.1210/er.2008-0002)

39 Frisen L, Nordenstrom A, Falhammar H, Filipsson H, Holmdahl G, Janson PO, Thoren M, Hagenfeldt K, Moller A \& Nordenskjold A. Gender role behavior, sexuality, and psychosocial adaptation in women with congenital adrenal hyperplasia due to CYP21A2 deficiency. Journal of Clinical Endocrinology and Metabolism 2009 94 3432-3439. (doi:10.1210/jc.2009-0636)

40 van Rossum EF \& van den Akker EL. Glucocorticoid resistance. Endocrine Development 201120 127-136.

41 Speiser PW, Azziz R, Baskin LS, Ghizzoni L, Hensle TW, Merke DP, Meyer-Bahlburg HF, Miller WL, Montori VM, Oberfield SE et al. Congenital adrenal hyperplasia due to steroid 21-hydroxylase deficiency: an Endocrine Society clinical practice guideline. Journal of Clinical Endocrinology and Metabolism 201095 4133-4160. (doi:10.1210/jc.2009-2631)

Received 2 October 2012

Revised version received 28 November 2012

Accepted 4 December 2012 